\title{
La resiliencia en adolescentes con problemas de la conducta, con un enfoque de género en estudiantes del $10^{\mathrm{mo}}$ grado
}

\author{
Resilience in adolescents with behavioral problems, with a gender focus on $10^{\text {th }}$ grade \\ students
}

\section{La resiliencia en adolescentes con problemas de la conducta}

\author{
Anicia Katherine Tarazona Meza(1) \\ Inger Solange Maitta Rosado ${ }^{(2)}$ \\ María José Intriago Basurto ${ }^{(3)}$ \\ (1) Universidad Técnica de Manabí, Portoviejo, Ecuador. email: atarazona@utm.edu.ec \\ (2) Universidad Técnica de Manabí, Portoviejo, Ecuador. email: imaitta@utm.edu.ec \\ (3) Universidad Técnica de Manabí, Portoviejo, Ecuador. email: mintriago7967@utm.edu.ec
}

Contacto: atarazona@utm.edu.ec.

Recibido: 18-02-2020 Aprobado:01-05-2020

\begin{abstract}
Resumen
La resiliencia consiste en un conjunto de atributos o habilidades innatas para afrontar adecuadamente situaciones adversas, como son factores estresantes y situaciones riesgosas. En los últimos años se discute con interés sobre la capacidad de reacción que pueden desarrollar algunos adolescentes cuando están expuestos a situaciones difíciles o agresiones. Algunas veces logran sobrepasar niveles de resistencia y terminan con más energía protectora que antes de la exposición a las situaciones adversas. El objetivo del trabajo consiste en ofrecer una caracterización de adolescentes que estudian en el nivel 10mo grado de educación básica, influenciados con problemas de conducta desde un enfoque de género, en unidades educativas públicas y privadas de Portoviejo. Para ello se parte de un método que incluye una amplia revisión bibliográfica direccionada al análisis de adolescentes de ambos sexos que confrontan problemas de conducta, mediante la aplicación de una investigación cualitativa transversal utilizando la escala SV-RES creada por los doctores Eugenio Saavedra y Villalta Marcos, que ofrece los factores determinantes en adolescentes que estudian el 10 mo grado en tres instituciones del sistema de educación, a decir el Colegio Particular Cruz del Norte, Colegio Particular Tecnológico Manabí y Colegio Fiscal Portoviejo. El trabajo permite concluir que los adolescentes del sexo femenino son ligeramente más resilientes que los del sexo masculino, así como el nivel mostrado por los estudiantes encuestados demuestra que, se
\end{abstract}

encuentran en condiciones de revertir los problemas de conducta que están confrontando.

Palabras clave: Adolescentes; adversidad; conducta; factores estresantes; género

\begin{abstract}
Resilience consists of a set of innate attributes or abilities to adequately cope with adverse situations, such as stressors and risky situations. In recent years, we discuss with interest the reaction capacity that some adolescents can develop when they are exposed to difficult situations or aggressions. Sometimes they manage to overcome resistance levels and end up with more protective energy than before exposure to adverse situations. The objective of the work is to offer a characterization of resilience in adolescents studying at the 10th grade level of basic education, influenced by behavior problems from a gender perspective, in public and private educational units of Portoviejo. This is based on a method that includes an extensive literature review aimed at the analysis of the resilience of adolescents of both sexes who face behavioral problems, through the application of a qualitative transversal research using the SV-RES scale created by doctors Eugenio Saavedra and Villalta Marcos, which offers the determinants of resilience in adolescents who study 10th grade in three institutions of the education system, namely the Cruz del Norte Private School, Manabí Technological Private School and Portoviejo Fiscal College. The work allows us to conclude that female adolescents are
\end{abstract}


resilient than male adolescents, as well as the level of resilience shown by the surveyed students shows that they are in a position to reverse the behavioral problems they are facing.

Keywords: Teenagers; adversity; conduct; stressors; gender

\section{Introducción}

La problemática de la conducta escolar se palpa todos los días en los hogares y aulas de clase. Los padres y docentes deben apelar a las estrategias pre establecidas por profesionales capacitados que, propicien la modulación de los comportamientos negativos o conductas consideradas como no adecuadas por los adolescentes (Zhinin, 2018).

La violencia en el ámbito escolar emerge regularmente de los problemas de conducta. El sistema educativo tradicional la ha venido enfrentando con castigos y métodos violentos en la relación del proceso de enseñanza-aprendizaje (UNICEF, 2017).La disciplina positiva como una alternativa innovadora, puede ayudar a dejar de lado las prácticas de la educación tradicional que ya no surten efecto en los estudiantes y propende al desarrollo de sanas relaciones afectivas que facilitan un clima de armonía y respeto mutuo en el aula (Jiménez, 2018). La modulación del comportamiento en los estudiantes de la Educación General Básica (EGB) del Ecuador, tiene como fin formular estrategias que el docente considere $\mathrm{y}$ aplique en su quehacer educativo para modular el comportamiento y mejorar la relación con los estudiantes (Jiménez, 2018).

La comunidad educativa debe comprometerse a disciplinar positivamente a los estudiantes, dejando a un lado los obsoletos métodos tradicionales y promoviendo la búsqueda de soluciones a los problemas de comportamiento en el contexto escolar.

La existencia de estudiantes con problemas de conducta en la EGB ecuatoriana, requiere potenciar la investigación de la problemática. Durante los últimos años la atención se ha concentrado en la influencia de la resiliencia en diferentes niveles y contextos educativos, ya sea para explicar el éxito o fracaso académico o bien para identificar los factores de riesgo que pueden influir en conductas desadaptadas o en los factores de protección que desarrollan algunos individuos o colectividades, para hacer frente a condiciones de vulnerabilidad o de alto riesgo.

Sin lugar a dudas las experiencias del tratamiento y gestión de la resiliencia en interés de resolver problemas sociales, ha sido motivo de la atención de diversos círculos científicos a nivel internacional (Del Valle, Ponce, Camus \& Álvarez 2017). Un considerable número de investigaciones desarrolladas durante los últimos años, han focalizado su atención en los distintos contextos educacionales.

Al considerar el escenario de controversia en torno a los resultados obtenidos a través de diferentes estudios, el propósito del trabajo radica en profundizar en la temática de la resiliencia en estudiantes de la EGB, comparando los factores de resiliencia desde el ángulo de género.

\section{Materiales y métodos}

La investigación es de tipo descriptivacomparativa, pues se describe el nivel resiliente de los estudiantes de 10 mo grado de la EGB de tres unidades educativas ubicadas en la ciudad de Portoviejo y se realiza la comparación de los factores de resiliencia presentes en estudiantes de ambos sexos. El diseño obedece a una investigación cualitativa, no experimental, orientada a la recolección de los datos en un solo momento, permitiendo la descripción de la realidad, el análisis de las variables y su posible incidencia e interrelación, según factores, áreas y/o dimensiones de interés del fenómeno estudiado.

El enfoque cualitativo transversal se puso en práctica durante la realización de la encuesta al determinar resultados de diferentes tipos en distintos niveles, respondiendo a la tradición de estudio de caso explicando, describiendo y explorando la información sobre los factores determinantes de la resiliencia en adolescentes que estudian el 10mo grado en las Unidades Educativas: Particular "Cruz del Norte", Particular "Tecnológico Manabí", Fiscal Portoviejo.

La población objeto de estudio es de 370 estudiantes. La muestra seleccionada corresponde a 77 estudiantes con problemas de conducta.

Dentro de las técnicas se aplicó la observación tanto la directa como la indirecta, así como la encuesta para la obtención de los datos. Como instrumentos se utilizaron la guía de observación y el cuestionario de la encuesta. Para recolectar los datos se aplicó la escala de resiliencia para jóvenes y adultos SV-RES, la que cuenta con índices favorables de confiabilidad y validez (Saavedra \& Villalta, 2008a).

La escala se constituye a partir de las fuentes interactivas sobre las que el sujeto se apropia de la realidad a través del habla, abriendo la posibilidad al comportamiento saludable o de superación de los eventos traumáticos. Las fuentes interactivas se 
pueden clasificar en tres categorías (Saavedra \& Villalta, 2008b): 1) Yo tengo (apoyo que la persona cree que puede recibir); 2) Yo soy...yo estoy (fortalezas intrapsíquicas y condiciones internas de la persona); 3) Yo puedo (habilidades de las personas para relacionarse y resolver problemas)

Además, la escala se integra sobre la base del modelo emergente de estudios de caso, en el que se consideran elementos tales como: que la respuesta resiliente es una acción orientada a metas, con respuestas sustentadas o vinculadas; posee una visión abordable del problema como conducta recurrente; tiene una visión de sí mismo caracterizada por elementos afectivos y cognitivos positivos o proactivos ante los problemas, y; posee las condiciones de base, es decir, un sistema de creencias y vínculos sociales que impregnan la memoria de seguridad básica y que de modo recursivo interpreta la acción específica y los resultados (Saavedra, 2003).
La escala de resiliencia SV-RES describe 12 factores de resiliencia, los que se agrupan en 4 ámbitos de profundidad. Al propio tiempo los 12 factores permiten describir las interacciones del sujeto: en primer lugar, consigo mismo, en segundo lugar, con los otros y en tercer lugar con sus posibilidades, es decir, con lo que él cree que puede hacer, según se expresa en la tabla 1.

La Escala de resiliencia SV-RES se integra con 60 ítems, los cuales se responden en una escala Likert de 5 grados, cuyas puntuaciones van de 1 (muy en desacuerdo) a 5 (muy de acuerdo). El instrumento presenta una adecuada validez $(\mathrm{r}=0,76)$ y un alto nivel de confiablidad $(\alpha=0,96)$ (Saavedra \& Villalta, 2008b). De acuerdo a ella, los participantes serán clasificados en cada uno de los factores de acuerdo a 3 categorías: alto; promedio y; bajo, dependiendo del percentil de posición respecto del baremo presentado por (Saavedra \& Villalta, 2008b), según se expone en la tabla 2.

\begin{tabular}{|l|l|l|l|l|}
\hline & $\begin{array}{l}\text { Condiciones de } \\
\text { base }\end{array}$ & $\begin{array}{l}\text { Visión de sí } \\
\text { mismo }\end{array}$ & Visión del problema & $\begin{array}{l}\text { Respuesta } \\
\text { resiliente }\end{array}$ \\
\hline $\begin{array}{l}\text { Yo soy, yo } \\
\text { estoy }\end{array}$ & F1. Identidad & F2. Autonomía & F3. Satisfacción & F4. Pragmatismo \\
\hline Yo tengo & F5. Vínculos & F6. Redes & F7. Modelo & F8. Metas \\
\hline Yo puedo & F9. Efectividad & F10. Autoeficacia & $\begin{array}{l}\text { F11. } \\
\text { Aprendizaje }\end{array}$ & $\begin{array}{l}\text { F12. } \\
\text { Generatividad }\end{array}$ \\
\hline
\end{tabular}

Tabla 1. Factores de resiliencia

Fuente: Elaboración propia a partir de la información de (Saavedra \& Villalta, 2008b)

La aplicación de la escala se realizó de modo directo y grupal, entregándole a cada participante el instrumento escala de resiliencia SV-RES, resaltando la voluntariedad al momento de contestar y la confidencialidad de los datos, cumpliendo con los requerimientos éticos para este tipo de estudio. El tiempo de respuesta fue de aproximadamente 20 minutos. Para el análisis se realizó un examen descriptivo sobre la base de distribuciones de frecuencia, medidas de tendencia central y de dispersión. Para el análisis comparativo se aplicó la prueba estadística no paramétrica de comparación de grupos U de Mann Whitney (Berlanga \& Rubio, 2012). El propósito consiste en identificar la influencia del género en los niveles de resiliencia que presentan los estudiantes.

\begin{tabular}{|l|l|}
\hline Percentil & Clasificación \\
\hline $0-25$ & Bajo \\
\hline $26-75$ & Promedio \\
\hline $76-100$ & Alto \\
\hline
\end{tabular}

Tabla 2. Interpretación del puntaje de los factores de resiliencia

Fuente: Elaboración propia en base a información obtenida de (Saavedra \& Villalta, 2008b)

\section{La resiliencia y la conducta en la escuela}

El concepto de resiliencia se viene estudiando y tomando fuerza en el debate científico desde la segunda mitad del Siglo XX; tiene como origen etimológico la palabra latina resilio que significa volver atrás, volver de un salto, resaltar, rebotar (Luthar, 2006).
En el campo de la Psicología, el término cobra importancia a través de los trabajos de quien considera que la resiliencia no debe ser entendida como la animada negación de las difíciles experiencias de la vida, dolores y cicatrices, siendo más bien, la habilidad para continuar adelante.

En síntesis, la resiliencia puede transformar o fortalecer a aquellas personas enfrentadas a la 
adversidad, manteniendo conductas de adaptación, permitiendo un desarrollo normal o promoviendo un crecimiento más allá del nivel de funcionamiento presente. Así entonces, se puede identificar como resiliencia en los estudiantes con problemas de conducta, a la capacidad de éstos para superar las dificultades y evitar resultados negativos, en términos conductuales, psicológicos, académicos, e incluso, físicos.

El comportamiento de los adolescentes en la escuela ha sido siempre una constante preocupación y tema que ha dado lugar a numerosas investigaciones y publicaciones. Resulta común que los diferentes actores se lancen acusaciones de culpabilidad. Muchos docentes señalan a los padres y a la pérdida de valores familiares como causa de una conducta escolar desajustada. Los padres de los jóvenes recriminan a la escuela y a los maestros de haber abandonado la mística educativa y de no comprender a la juventud. Ambos, padres y educadores, acusan a la sociedad con sus medios de comunicación, sus atractivos perniciosos e incluso a sus leyes de protección al menor de ser en buena parte responsables del descontrol de la juventud. Esta, por su parte, rechaza una vida familiar y escolar que pretende fundamentarse en patrones de relación que consideran autoritarios y no acordes con la realidad actual (Cajamarca, 2018).

El discurso de los mayores sobre los supuestos valores que se deben practicar, no les significa mucho en un mundo en el que ven a estos mismos adultos dejarlos de lado cada vez que les conviene. Por otra parte, tanto a nivel familiar como escolar, los adultos se quejan de que ya los jóvenes no se someten a la autoridad como sucedía en generaciones anteriores.

Es común la afirmación de que tiempos pasados eran mejores y que en el mundo de hoy algo se ha perdido, razón por la cual la juventud anda como anda. Este algo que se ha perdido se refiere generalmente, al poder del adulto para someter a los hijos o a los estudiantes a una obediencia estricta y sin discusión posible. Tal sometimiento se resume en una frase que se escucha muy a menudo, de boca de quienes añoran esas épocas, en las que todo supuestamente, estaba derecho y en su lugar, 2014).

La alianza de padres y maestros en el sometimiento de los niños y jóvenes adolescentes, es otro aspecto de esos tiempos idílicos que echan de menos algunos. Entonces por ejemplo dicen que: si se enviaba una queja a los padres del mal comportamiento de un determinado estudiante, este recibía una reprimenda o una golpiza en su casa. Ahora, en estos casos, los padres la toman contra el maestro o la escuela. Las cosas han cambiado y para los que piensan así, por supuesto que han cambiado para mal.

Pero lo que, si queda claro, siendo justos con la historia, es que, en las generaciones de los padres, los abuelos o los bisabuelos de los estudiantes de hoy, los conflictos escolares entre estudiantes y docentes, entre compañeros de clases o entre padres y escuela, siempre han existido, aunque con matices diferentes dados el influjo propio de las sociedades pasadas. Por lo que pensar que, el proceso de enseñanza-aprendizaje corría sobre rieles y sin grandes problemas, corresponde a un enfoque que aprecia el problema de manera superficial, arbitraria y vertical.

Tradicionalmente, se les ha estado exigiendo a los estudiantes un mismo tipo de atención, de compostura o de compromiso con los deberes. Estar sentados por cuarenta o cuarenta y cinco minutos, no hablar mientras un maestro o profesor dicta una clase, no perder la atención sin que importe que la lección esté resultándole muy aburrida, que sean capaces de soportar las ganas de aliviar sus necesidades corporales hasta terminada la clase, que nunca se peleen entre ellos, que no se molesten unos a otros, etc. Tales exigencias son paradójicas, pues no toman para nada en cuenta las características evolutivas del desarrollo humano.

La discusión debe centrarse en las conductas que realmente puede afectar la estabilidad de la colectividad y la buena marcha del proceso docente educativo, entre las que se pueden señalar las siguientes (Morales \& González, 2014): todas aquellas que van en detrimento de la integridad física o psicológica del mismo alumno, de sus compañeros o de otras personas; las que perjudican las pertenencias materiales de los demás estudiantes o las instalaciones o equipos de la escuela; todo comportamiento que perturbe la atención y concentración en una tarea o lección, tanto de quien las ejecuta como de los demás alumnos; los actos de irresponsabilidad como negarse a cumplir con sus obligaciones académicas, ya sea no haciendo las tareas encomendadas o abandonar las clases sin permiso de padres o docentes.

\section{Resultados}

La investigación se realizó con un grupo de estudiantes que fueron seleccionados para la muestra en tres instituciones educativas radicadas en la ciudad de Portoviejo: el Colegio Particular Cruz del Norte; el Colegio Particular Manabí Tecnológico y; el Colegio Fiscal Portoviejo. 
En la tabla 3 se exponen las características de la planta docente educativa de los colegios estudiados.
En la figura 1 se muestra el comportamiento gráfico de la distribución por género de la muestra seleccionada por colegios.

\begin{tabular}{|c|c|c|c|c|c|c|}
\hline \multirow{2}{*}{ Unidad educativa } & \multirow{2}{*}{$\begin{array}{l}\text { Total } \\
\text { docentes }\end{array}$} & \multirow{2}{*}{$\begin{array}{l}\text { Total de } \\
\text { estudiantes }\end{array}$} & \multirow{2}{*}{$\begin{array}{l}\text { De ellos en } \\
\text { grado } 10^{\text {mo }}\end{array}$} & \multirow{2}{*}{ Muestra } & \multicolumn{2}{|l|}{ Por sexos } \\
\hline & & & & & Masculino & Femenino \\
\hline Colegio Particular Cruz del Norte & 27 & 395 & 60 & 13 & 7 & 6 \\
\hline Colegio Particular Manabí Tecnológico & 46 & 1146 & 97 & 31 & 17 & 14 \\
\hline Colegio Fiscal Portoviejo & 195 & 4374 & 252 & 33 & 18 & 15 \\
\hline TOTAL & 268 & 5915 & 409 & 77 & 42 & 35 \\
\hline
\end{tabular}

Tabla 3. Características generales de la planta docente educativa por unidades

Fuente: Elaboración propia con información obtenida de: https://www.infoescuelas.com/ecuador/manabi

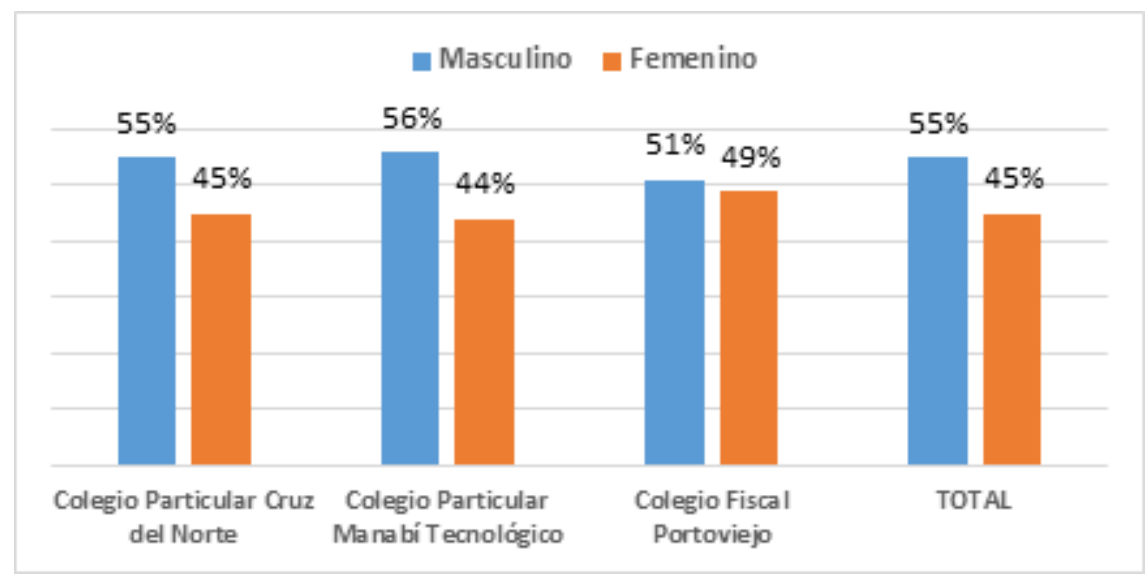

Figura 1. Comportamiento gráfico de la distribución por género

Fuente: Elaboración propia con información obtenida de: https://www.infoescuelas.com/ecuador/manabi

\section{Resultados de la aplicación de la escala de resiliencia $S V$-RES}

En la tabla 4 se muestran los resultados de la aplicación de la escala de resiliencia a la muestra de estudiantes de las instituciones educativas seleccionadas, en función de los 12 factores resilientes por género.

Cuando se realiza el análisis de los datos expuestos anteriormente se pueden apreciar las siguientes particularidades por género:
En el factor identidad el $6 \%$ de los estudiantes califica con un valor alto, mostrando un incremento los varones; el $61 \%$ califica con una puntuación de promedio, siendo ligeramente más elevado en las hembras y; el $33 \%$ con un valor bajo, resultando ligeramente superior en las hembras. En el factor autonomía el $11 \%$ de los estudiantes califica con un valor alto, mostrando un ligero incremento las hembras; el $56 \%$ califica con una puntuación de promedio, siendo más elevado en los varones $\mathrm{y}$; el $33 \%$ con un valor bajo, resultando ligeramente superior en las hembras. 


\begin{tabular}{|c|c|c|c|c|c|c|c|c|c|c|c|c|c|c|c|c|c|c|}
\hline \multirow[b]{3}{*}{ Dimensiones } & \multicolumn{6}{|c|}{ Alto } & \multicolumn{6}{|c|}{ Promedio } & \multicolumn{6}{|c|}{ Bajo } \\
\hline & \multicolumn{2}{|c|}{ Masculino } & \multicolumn{2}{|c|}{ Femenino } & \multicolumn{2}{|c|}{ Total } & \multicolumn{2}{|c|}{\begin{tabular}{|l|} 
Masculino \\
\end{tabular}} & \multicolumn{2}{|c|}{ Femenino } & \multicolumn{2}{|c|}{ Total } & \multicolumn{2}{|c|}{ Masculino } & \multicolumn{2}{|c|}{ Femenino } & \multicolumn{2}{|c|}{ Total } \\
\hline & Frec. & $\%$ & Frec. & $\%$ & Frec. & $\%$ & Frec. & $\%$ & Frec. & $\%$ & Frec. & $\%$ & Frec. & $\%$ & Frec. & $\%$ & Frec. & $\%$ \\
\hline Identidad & 1 & 10 & 0 & 0 & 1 & 6 & 6 & 60 & 5 & 63 & 11 & 61 & 3 & 30 & 3 & 38 & 6 & 33 \\
\hline Autonomía & 1 & 10 & 1 & 13 & 2 & 11 & 6 & 60 & 4 & 50 & 10 & 56 & 3 & 30 & 3 & 38 & 6 & 33 \\
\hline \begin{tabular}{|l|} 
Satisfacción \\
\end{tabular} & 0 & 0 & 1 & 13 & 1 & 6 & 5 & 50 & 5 & 63 & 10 & 56 & 5 & 50 & 2 & 25 & 7 & 39 \\
\hline Pragmatismo & 1 & 10 & 1 & 13 & 2 & 11 & 5 & 50 & 4 & 50 & 9 & 50 & 4 & 40 & 3 & 38 & 7 & 39 \\
\hline Vínculos & 0 & 0 & 2 & 25 & 2 & 11 & 6 & 60 & 5 & 63 & 11 & 61 & 4 & 40 & 1 & 13 & 5 & 28 \\
\hline Redes & 1 & 10 & 1 & 13 & 2 & 11 & 5 & 50 & 5 & 63 & 10 & 56 & 4 & 40 & 2 & 25 & 6 & 33 \\
\hline Modelos & 1 & 10 & 1 & 13 & 2 & 11 & 5 & 50 & 4 & 50 & 9 & 50 & 4 & 40 & 3 & 38 & 7 & 39 \\
\hline Metas & 0 & 0 & 0 & 0 & 0 & 0 & 6 & 60 & 5 & 63 & 11 & 61 & 4 & 40 & 3 & 38 & 7 & 39 \\
\hline Efectividad & 0 & 0 & 0 & 0 & 0 & 0 & 5 & 50 & 5 & 63 & 10 & 56 & 5 & 50 & 3 & 38 & 8 & 44 \\
\hline Autoeficacia & 0 & 0 & 0 & 0 & 0 & 0 & 6 & 60 & 4 & 50 & 10 & 56 & 4 & 40 & 4 & 50 & 8 & 44 \\
\hline Aprendizaje & 0 & 0 & 1 & 13 & 1 & 6 & 5 & 50 & 5 & 63 & 10 & 56 & 5 & 50 & 2 & 25 & 7 & 39 \\
\hline \begin{tabular}{|l|} 
Generatividad \\
\end{tabular} & 0 & 0 & 0 & 0 & 0 & 0 & 6 & 60 & 5 & 63 & 11 & 61 & 4 & 40 & 3 & 38 & 7 & 39 \\
\hline
\end{tabular}

Tabla 4. Resultados de la aplicación de la escala de resiliencia

Fuente: Elaboración propia a partir de los datos obtenidos mediante la aplicación de la

\section{escala SV-RES}

El factor satisfacción el $6 \%$ de los estudiantes califica con un valor alto, mostrando un incremento en las hembras; el 56\% califica con una puntuación de promedio, siendo ligeramente más elevado en las hembras y; el $39 \%$ con un valor bajo, resultando ligeramente superior en los varones. En el factor pragmatismo el $11 \%$ califica con un valor alto, mostrando un ligero incremento las hembras; el $50 \%$ con una puntuación de promedio, y; el 39\% con un valor bajo, resultando ligeramente superior en los varones.

En la figura 2 se muestra el comportamiento gráfico de los 12 factores resilientes por género.

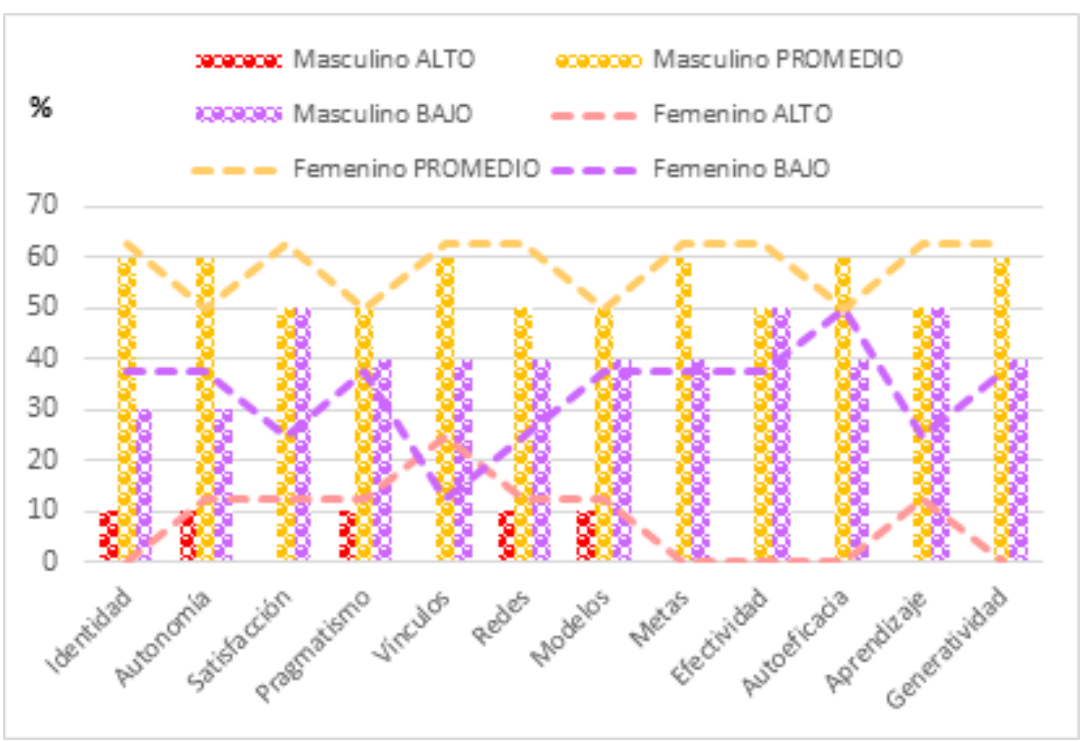

Figura 2. Comportamiento gráfico de los 12 factores resilientes por género

Fuente: Elaboración propia a partir de los datos obtenidos mediante la aplicación de la escala SV-RES

El factor vínculos el $11 \%$ de los estudiantes califican con un valor alto, dominado por el sexo femenino; el $61 \%$ califica con una puntuación de promedio, con un ligero incremento en las hembras y; el $28 \%$ con un valor bajo, resultando superior en los varones. En el factor redes el $11 \%$ califica con un valor alto, con un ligero incremento en las hembras; el $56 \%$ con un valor promedio, siendo más elevado en las hembras y; el $33 \%$ califica con un valor bajo, resultando superior en los varones.
En el factor modelos el $11 \%$ califica con un valor alto, resultando ligeramente superior en las hembras; el $50 \%$ califica con un valor promedio para ambos sexos; mientras que $39 \%$ califica con un valor bajo, con un ligero incremento en los varones. En el factor metas ningún estudiante calificó con un valor alto; el $61 \%$ califica con un valor promedio, resultando ligeramente más elevado en las hembras y; 39\% con valores bajos, siendo más alto en los varones. 
En el factor afectividad ningún estudiante califica con valor alto; el $56 \%$ corresponde a valores promedio, resultando ligeramente superior en las hembras y; el $44 \%$ con valores bajos, siendo más elevado en los varones. En el factor ningún estudiante califica con valor alto; el $56 \%$ califica con un valor promedio, siendo ligeramente más elevado en los varones y; el $44 \%$ califica con un valor bajo, resultando superior en las hembras.

En el factor aprendizaje el $6 \%$ de los estudiantes calificó con una puntuación alta, correspondiendo en su totalidad a las hembras; el $56 \%$ califica con un valor promedio, resultando más elevado en las hembras; mientras que el $39 \%$ califica con un valor bajo, siendo más elevado en los varones. En el factor generatividad ningún estudiante califica con valor alto; el $61 \%$ califica con una puntuación promedio, siendo más elevado en las hembras y; el $39 \%$ como bajo, siendo más elevado en los varones.

En la figura 3 se muestra la evaluación gráfica del nivel de resiliencia por género.

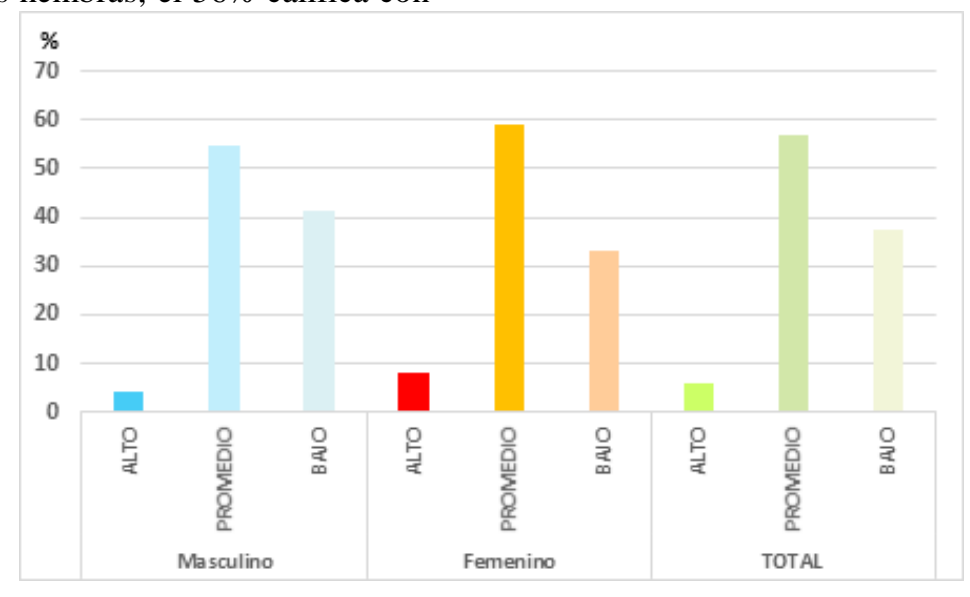

Figura 3. Evaluación gráfica del nivel de resiliencia por género

Fuente: Elaboración propia a partir de los datos obtenidos mediante la aplicación de la escala SV- RES

Se puede apreciar de manera general que el $62 \%$ de los estudiantes califica con un nivel de resiliencia entre alto y promedio, mientras que el $38 \%$ con un nivel bajo. Las hembras califican ligeramente por encima de la media con el $66 \%$ entre alto y promedio.

\section{Discusión}

En Ecuador son varios los autores que han incursionado en el estudio de los problemas de conducta y su influencia en el aprovechamiento académico (Freire, 2014; Cueva, 2015; Yagual, 2016; Guachamin, 2017; Cajamarca, 2018). La provincia de Manabí no se ha quedado rezagada y cuanta con diversos resultados investigativos asociados a los problemas de conducta de los estudiantes y su influencia en el aprovechamiento académico (Alcívar, 2016; Mendoza, \& Mestre, 2018; Vera, Triviño, Briones, 2018; Romero \& Lino, 2018; Segovia, 2018; Loor, Moreira \& Arroyo, 2019). De manera general los resultados de las investigaciones realizadas demuestran que a pesar del contexto social y territorial, los problemas más comunes que se presentan están asociados a la violencia, el aprovechamiento académico y la disciplina, así como, se pudo comprobar que los estudiantes que presentan características resilientes se incorporan con mayores niveles de adaptación e integración a los distintos sistemas educativos, independiente de los contextos de vulnerabilidad que se puedan presentar (Saavedra 2003; Gaxiola, González, Contreras \& Gaxiola, 2012; Morales \& González, 2014; Rodríguez, Guzmán, \& Yela, 2012).

Cuando se analizan los resultados de los trabajos desarrollados por otros autores en relación con la presente investigación se puede observar, que no se poseen evidencias concluyentes respecto de la relación entre resiliencia y género y que las diferencias en la puntuación total de los factores medidos entre hombres y mujeres, no evidencian resultados estadísticamente significativos (Saavedra, Castro \& Inostroza, 2012), pero si se han identificado diferencias en el área de la interacción y en el área del apoyo social.

Una de las cuestiones que sobresale en el trabajo derivado de la aplicación de la escala de resiliencia, es la posibilidad de adaptación al patrón cultural ecuatoriano y especialmente al de la provincia de Manabí. El instrumento se enfocó a los estudiantes, permitiendo medir el nivel de resiliencia de manera 
general, además de conocer los 12 factores específicos que lo conforman y poder trabajar sobre ellos.

\section{Conclusiones}

El trabajo permitió ofrecer una caracterización de la resiliencia en adolescentes que estudian en el nivel 10mo grado de educación básica, influenciados con problemas de conducta desde un enfoque de género, en unidades educativas públicas y privadas de Portoviejo, pudiendo apreciar que la mayoría de los estudiantes encuestados demuestran ser capaces de establecer juicios generales que tienen su origen en los valores culturales que lo definen, con capacidad de reflexión, definiendo su aporte particular a la sociedad, develando la manera particular de interpretar los problemas surgidos y las acciones personales que realizan para resolverlos.

Se ha podido verificar que la mayoría de los estudiantes demostraron ser capaces de valorar la socialización con el colectivo a partir de su historia personal, mediante el establecimiento de vínculos afectivos con las personas de su entorno social, donde las redes sociales pueden jugar un papel importante para la solución y superación de las situaciones problemáticas que surgen, demostrando capacidad para establecer juicios referidos al valor contextual de metas y redes sociales sobre la situación problemática.

La caracterización de la resiliencia en adolescentes demostró que los estudiantes pueden establecer juicios relacionados con las posibilidades propias y el vínculo con el entorno, focalizadas en obtener el éxito a partir de los propios esfuerzos y la colaboración del colectivo, siendo capaces de apreciar los problemas como una oportunidad para el engrandecimiento y la superación personal, para lo cual se reconoce la posibilidad de solicitar ayuda a otros.

Se pudo verificar mediante los resultados de la investigación, que la gestión de la resiliencia constituye una actividad clave para lograr focalizar los esfuerzos en el mejoramiento de los resultados académicos de los estudiantes, por cuanto permite establecer una diferenciación de la capacidad de los alumnos, para superarse a partir de sus propias experiencias, aun en situación de desventaja, donde sobresale la posibilidad de aprovechar las potencialidades de los colectivos y las redes en el enfrentamiento y superación de las situaciones problémicas.

Independientemente de que los resultados del trabajo demuestran que existe un nivel resiliente ligeramente superior en las hembras, no se puede afirmar de manera concluyente que, exista una relación entre resiliencia y género; pero que en este caso las diferencias principales se localizan en los factores: satisfacción, vínculos, redes, afectividad, aprendizaje y generatividad, donde las hembras ofrecen resultados más elevados que los varones.

\section{Bibliografía}

Alcivar, M. (2016). Estrategia de sensibilización sobre violencia intrafamiliar para mejorar la conducta de los niños y niñas dela Unidad Educativa Colón Arteaga García de la parroquia Ricaurte, cantón Chone. Trabajo de titulación previo para la obtención del título de Psicóloga Clínica. Universidad Laica Eloy Alfaro de Manabí. Extensión Chone.

Berlanga, V. \& Rubio, M. (2012). Clasificación de pruebas no paramétricas. Cómo aplicar las en SPSS. REIRE, 5(2), 101-113.

Cajamarca, M. (2018). Causas que inciden en la conducta y el bajo rendimiento académico de los niños del séptimo A de la escuela de educación general básica Jesús Vázquez Ochoa del cantón Cuenca, en el año lectivo 2017-2018. Trabajo de titulación previo a la obtención del título de Licenciada en Educación. Universidad Politécnica Salesiana de Cuenca.

Cueva, L. (2015). Análisis de los factores socioeconómicos y su impacto en el rendimiento académico de los niños y niñas de la escuela "Dr. Daniel Rodas" de la ciudad de Loja. Tesis de grado para la obtención del título de Economista. Universidad Internacional del Ecuador - Loja. Facultad de Ciencias Administrativas y Económicas.

Del Valle, Milenko. Ponce, Nicolás. Camus, Pablo. Álvarez, Gabriel. (2017). Factores resilientes en estudiantes vulnerables. El Caso de los Programas Propedéutico y CEIES de la Universidad de Antofagasta. INNOVARE, revista electrónica de educación superior. ISSN: 0719-7500.

Freire, D. (2014). La conducta disruptiva y su incidencia en el rendimiento académico de las/os estudiantes de los 10 mos grados de educación general básica paralelos "I, II" del Colegio Universitario "Juan Montalvo", de la provincia de Tungurahua, cantón 
Ambato. Informe final del Trabajo de Titulación. Universidad Técnica de Ambato. Facultad de Ciencias Humanas y de la Educación. Carrera de Psicología Educativa.

Gaxiola, J. González, S. Contreras, Z. \& Gaxiola, E. (2012). Predictores del rendimiento académico en adolescentes con disposiciones resilientes y no resilientes. Revista de psicología, 30(1), 47-74.

Guachamin, M. (2017). Influencia de la adaptación escolar en el rendimiento académico de los estudiantes de octavo año de educación general básica del Colegio Luciano Andrade Marín de la ciudad de Quito periodo 2016 -2017. Trabajo de titulación previo a la obtención del Título de Licenciada en Ciencias de la Educación. Universidad Central del Ecuador. Facultad de Filosofía, Letras y Ciencias de la Educación. Carrera de Psicología Educativa y Orientación Vocacional.

Jiménez, María. (2018). Disciplina positiva y la modulación del comportamiento de estudiantes de educación general básica en el Ecuador. Universidad Andina Simón Bolívar, sede Ecuador, área de Educación. Trabajo previo presentado en la maestría en Innovación de la Educación.

Loor, M. Moreira, M \& Arroyo, M. (2019). La disciplina y el rendimiento escolar de los estudiantes de educación general básica en la Unidad Educativa Club Rotario de Portoviejo. Revista Cognosis. VOL. 4 NÚM. 2 (2019): ABRIL-JUNIO / Artículos

Luthar, S. (2006). Resilience in development: A synthesis of research across five decades. In D. Cicchetti y D. J. Cohen (Eds.), Developmental Psychopathology: Risk, disorder and adaptation. New York: Wiley, 2006: p. 740-795.

Mendoza, C. \& Mestre, U. (2018). Incidencia de la consejería estudiantil de la reducción de problemas de conducta de los escolares en la Unidad Educativa fiscal gran Colombia de la ciudad de Portoviejo, Manabí, Ecuador. Revista Pertinencia Académica. ISSN 2588-1019, (8), 141-158. Disponible en: http://revistaacademica.utb.edu.ec/index.php/pertacade/ article/view/134.

Morales, M. \& González, A. (2014). ResilienciaAutoestima-Bienestar psicológico y capacidad intelectual de estudiantes de cuarto medio de buen rendimiento de liceos vulnerables. Estudios Pedagógicos, 40(1), 215-228.

Rodríguez, H. Guzmán, L. \& Yela, N. (2012). Factores personales que influyen en la resiliencia en niños y niñas en edades comprendidas entre 7 y 12 años que se desarrollan en extrema pobreza. International Journal of psychological research, 5(2), 98-107.

Romero, S \& Lino, G. (2018). Conflictos familiares y su influencia en la disciplina de las y los estudiantes del noveno año de educación básica de la Unidad Educativa mixta Portoviejo, período 2017. Revista Atlante: Cuadernos de Educación y Desarrollo (agosto 2018). En línea: https://www.eumed.net/rev/atlante/2018/0 8/conflictos-familiares-estudiantes.html/

Saavedra, E. (2003). El Enfoque Cognitivo Procesal Sistémico, como posibilidad de Intervenir educativamente en la formación de sujetos Resilientes: estudio de casos. Tesis Doctoral, Universidad de Valladolid, España.

Saavedra, E., Castro, A. \& Insotroza, A. (2012). Niveles de resiliencia en adultos diagnosticados con y sin depresión. Revista Pequén, 2(1), 161-184.

Saavedra, E. \& Villalta, M. (2008a). Medición de las características resilientes, un estudio comparativo en personas entre 15 y 65 años. Liberabit, 14(14), 31-40.

Saavedra, E., \& Villalta, M. (2008b). Escala de resiliencia SV-RES para jóvenes y adultos. Santiago: CEANIM.

Segovia, M. (2018). Factores psicosociales en el desempeño estudiantil de la escuela Ocho de Enero del Cantón Jipijapa. Proyecto de investigación para la titulación de licenciada en enfermería. Universidad Estatal del Sur de Manabí. Facultad De Ciencias de la Salud. Carrera de Enfermería

UNICEF. (2017). Una mirada en profundidad al acoso escolar en el Ecuador. Fondo de las Naciones Unidas para la Infancia y el Ministerio de Educación del Ecuador. Consultado el 20 septiembre de 2019. Disponible en: https://www.unicef.org/ecuador/acoso_esc olar final002.pdf. 
Vera, L. Triviño, J. Briones, Y. (2018). Procedimientos implementados en la Unidad Educativa Fiscal Portoviejo, provincia de Manabí, Ecuador, para la resolución de conflictos entre estudiantes de 3er año de bachillerato. Revista Didasc@lia: D\&E. Publicación cooperada entre CEDUT- Las Tunas y CEdEGGranma, Cuba. Vol. IX. Año 2018. Número 4, octubre-diciembre.

Yagual, G. (2016). Análisis de problemas sociales y su influencia en el rendimiento académico Colegio Experimental José García Cando. Proyecto de investigación previo a la titulación en la Carrera de Organización y Desarrollo Comunitario. Universidad Estatal Península de Santa Elena.

Zhinin, Lidia. (2018). Causas que inciden en los problemas disciplinarios de niños y niñas del 5to A de la Escuela de Educación Básica Julio María Matovelle en el año lectivo 2017-2018. Universidad Politécnica Salesiana, sede Cuenca, Ecuador. Trabajo presentado para optar por el título de Licenciada en Ciencias de la Educación. 